\title{
Seroprevalence of Scrub Typhus, Spotted Fever, and Murine Typhus in Vellore District, Tamil Nadu
}

\author{
${ }^{1}$ Kalaimani Saravanan, ${ }^{1}$ Lisha Jha, ${ }^{1}$ John A J Prakash, ${ }^{2}$ Rajasekar Aruliah \\ and 'Ernest David* \\ 1Department of Clinical Microbiology, Christian Medical College, Vellore-632004. \\ 2Department of Biotechnology, Thiruvalluvar University, Serkadu, Vellore-632115.
}

Received: 16 Oct 2020 / Accepted: 14 Nov 2020/ Published online: 01 Jan 2021 *Corresponding Author Email: ernestdavid2002@yahoo.com

\begin{abstract}
Background: Rickettsial infections remain under-diagnosed and rarely reported due to limited data on seroprevalence and lack of diagnostic facilities in India. The aim of this study was to investigate the seroprevalence of Rickettsial infection in Vellore district, Tamilnadu. Methodology: A descriptive cross-sectional serosurvey was conducted from September 2017 to February 2018 in seven urban and seven rural areas of Vellore districts of Tamil Nadu. Serum samples were collected from 536 healthy individuals and tested by Enzyme-linked immunosorbent assay (ELISA) for Scrub typhus (ST), Spotted fever (SF), Murine typhus(MT) antibodies at the Christian Medical College (CMC), Vellore. Results: Of the 464 participants, 178 (33.2\%) were males and 356 (67\%) were female. The median age of participants was 42 years (IQR 31-54 years). The majority were either farmers or housewives. Exposure to rickettsial infection was observed in more than $1 / 3$ rd of the population surveyed. ST was the commonest among the three diseases surveyed. The seroprevalence of ST, SF, and MT was higher in rural areas than in urban areas. Seroprevalence of dual infection was also calculated and the commonest combination being ST and SFG infections $2.2 \%(12 / 536)$ followed by MT and SF infections $0.3 \%(2 / 536)$. Conclusion: Rickettsial infections are common in the Vellore district and prevalence is higher in rural than urban areas.
\end{abstract}

\section{Keywords}

Vellore; Scrub typhus; Spotted fever group rickettsiae; Typhus group rickettsiae

\section{INTRODUCTION:}

Rickettsial infections are caused by bacterial organisms (Rickettsiae), which are found throughout the world (1). They are caused by obligate intracellular Gram-negative bacteria of genera Rickettsiae and Orientia. The infections are transmitted to humans through bites of infected arthropod vectors, such as fleas, mites, ticks, lice and chiggers (2). The genus Rickettsia comprises more than 30 species and traditionally characterized into two main groups, the spotted fever group (SFG) and the typhus group (TG), which are the main established human pathogens (3).
R.rickettsii, R.conorii, R.prowazekii and R.typhi whic $h$ cause Rocky Mountain spotted fever (RMSF), Mediterranean spotted fever (MSF), epidemic and endemic typhus respectively are the important members of the genus Rickettsiae (4). The Orientia genus comprises two species; O.tsutsuga mushi and O.chuto together forming the (STG) scrub typhus group (5). SFG and TG rickettsiae infections have a worldwide distribution and are a significant cause of morbidity in Southeast Asia (6). STG was originally thought to be confined to be the AsiaPacific region but now has been reported from the Middle East, Africa, and South America(1). Rickettsioses are both emerging and re-emerging 
infections (7).In India, the rickettsial disease has been demonstrated from various parts of the country (1). The Vellore is in a tropical savanna climate at an average elevation of 288 meter $\{148-909$ meter $\}$ (8). The district covers an area of $6,075 \mathrm{~km} 2$ and a housing population of $3,936,331$ as reported by the 2011 census (9). The average minimum temperature ranges from $18.2^{\circ} \mathrm{C}\left(64.8^{\circ} \mathrm{F}\right)$ to $26.5^{\circ} \mathrm{C}\left(79.7^{\circ} \mathrm{F}\right)$ whereas the average maximum temperature range from $28.9^{\circ} \mathrm{C}\left(84^{\circ} \mathrm{F}\right)$ to $38.2^{\circ} \mathrm{C}\left(100.8^{\circ} \mathrm{F}\right)$. The humidity ranges from $40 \%-63 \%$ during summer and $67 \%-86 \%$ during winter. This data was obtained from http://www.imdchennai.gov.in/. This study was performed to determine the seroprevalence associated with scrub typhus, spotted fever, murine typhus in Vellore district in Tamil Nadu.

\section{METHODS:}

\section{Study population}

The descriptive cross-sectional serosurvey was carried out from September 2017 to February 2018. Seven (rural) villages and seven Urban (town) are in the Vellore district were selected for serosurvey. Study areas were randomly selected from a list that was derived based on 13 years (2005-2017) of scrub typhus data. The selected urban and rural areas are as depicted in Fig 1. In each area, one individual from one household was enrolled after obtaining informed consent. Clotted blood samples were collected in red-capped serum tube, (BD Vacutainer, Franklin Lakes, NJ, USA) from eligible, consenting adults ( $>18$ years old) who had no history of fever in the past 3 months.

\section{Laboratory analysis.}

The serum was separated by centrifuging in a refrigerated centrifuge (Eppendorf Centrifuge 5804 R, Eppendorf, Hamburg, Germany) at 3000 rpm for 10minutes at $\quad 4^{\circ} \mathrm{C}$. IgG antibodies to O.tsutsugamushi (56kDa antigens from Karp,
Kato, Gilliam, and TA716 strains) were detected in serum using the scrub typhus IgG ELISA (InBios International Inc., Seattle, WA). Murine typhus IgG antibodies were detected using R.typhi IgG ELISA (Fuller Laboratory, CA) which covers the speciesspecific protein rOmp B. For spotted fever IgG, we used the Rickettsia conorii IgG ELISA (Vircell, Granada, Spain) as per the manufacturer's instructions (14). All sera were tested at 1:100 dilution and an OD cutoff value 1.5 was considered positive as described previously (10).

Statistics: Data was entered into an Excel sheet (Excel 2016, Microsoft Corporation, Redmond, WA, USA). Median, percentage, and IQR using the Excel2016. Population data of the areas surveyed was obtained by accessing the Vellore census data (9). Seroprevalence was calculated using the online calculator developed and maintained by the Clinical and Translational Science Institute, UCSF (11), and uses the Reiczigel algorithm to calculate true prevalence (12).

\section{RESULTS:}

Serum samples of 536 adults from the Vellore district, 219 urban and 317 rural were subjected to IgG ELISA for detecting antibodies to Scrub typhus, murine typhus, and spotted fever. The median age of participants was 49 years (IQR 31-54 years), 178 were $(33.2 \%)$ males, and 356 (67\%) females. The most common occupation was a farmer (37\%) followed by housewives (35\%). Seroprevalence of ST, $\mathrm{SF}$, and MT is as shown in Fig 2, 3 and 4. There was a significant difference in the prevalence of these three diseases between the rural and urban populations studied as shown in Table 2. Prevalence in rural areas varies from $4 \%-56 \%(\mathrm{ST}), 3 \%-39 \%(\mathrm{SF})$, $1 \%-10 \%(\mathrm{MT})$ and in urban areas it was $3 \%-40 \%(\mathrm{ST})$, $2 \%-11 \%(\mathrm{SF}), 2 \%-5 \%(\mathrm{MT})$.

Table 1: Results of the sero-survey with cases expected in the study areas.

\begin{tabular}{|c|c|c|c|c|c|c|c|c|}
\hline \multirow{2}{*}{ Area } & \multirow{2}{*}{ Population } & \multirow{2}{*}{$\mathbf{n}$} & \multicolumn{3}{|c|}{ Sero-positive } & \multicolumn{3}{|c|}{ Expected cases in each area } \\
\hline & & & ST & SF & MT & ST & SF & MT \\
\hline Kaniyambadi * & 9597 & 31 & 1 & 2 & 0 & 310 & 619 & 310 \\
\hline Latteri * & 8522 & 34 & 8 & 4 & 2 & 2005 & 1003 & 501 \\
\hline Serkadu * & 3455 & 61 & 24 & 10 & 0 & 1359 & 566 & 57 \\
\hline Sirukanchi * & 1163 & 81 & 22 & 3 & 5 & 316 & 349 & 72 \\
\hline Thimiri * & 4939 & 33 & 14 & 1 & 0 & 2095 & 150 & 150 \\
\hline Ussoor* & 3248 & 29 & 2 & 0 & 0 & 224 & 112 & 112 \\
\hline Vadakadapanthangal * & 1111 & 48 & 12 & 2 & 4 & 278 & 46 & 93 \\
\hline Rural & 30924 & 317 & 83 & 20 & 11 & 6309 & 2799 & 1295 \\
\hline Alamelumangapuram & 7900 & 36 & 2 & 1 & 0 & 439 & 219 & 219 \\
\hline Bagayam & 9898 & 31 & 6 & 2 & 0 & 1916 & 319 & 319 \\
\hline K.V.Kuppam & 5321 & 23 & 7 & 1 & 0 & 1619 & 231 & 231 \\
\hline Kaspa & 4020 & 33 & 0 & 0 & 0 & 122 & 122 & 122 \\
\hline
\end{tabular}




\begin{tabular}{lllllllll}
\hline Thottapalayam & 6000 & 32 & 3 & 0 & 1 & 563 & 188 & 188 \\
Thuthipattu & 2880 & 34 & 10 & 3 & 0 & 847 & 254 & 85 \\
Arcot & 5955 & 30 & 1 & 0 & 0 & 199 & 199 & 199 \\
Urban & $\mathbf{4 1 9 7 4}$ & $\mathbf{2 1 9}$ & $\mathbf{2 9}$ & $\mathbf{7}$ & $\mathbf{1}$ & $\mathbf{5 7 0 5}$ & $\mathbf{1 5 3 2}$ & $\mathbf{1 3 6 3}$ \\
Total (Rural \& Urban) & $\mathbf{7 4 0 0 9}$ & $\mathbf{5 3 6}$ & $\mathbf{1 1 2}$ & $\mathbf{2 9}$ & $\mathbf{1 2}$ & $\mathbf{1 5 4 6 5}$ & $\mathbf{4 0 0 4}$ & $\mathbf{1 6 5 7}$ \\
\hline
\end{tabular}

Table 2: Prevalence of rickettsioses in Vellore district

\begin{tabular}{|c|c|c|c|c|c|c|}
\hline \multirow{2}{*}{ Area } & \multicolumn{2}{|c|}{ ST Prevalence } & \multicolumn{2}{|c|}{ SF Prevalence } & \multicolumn{2}{|c|}{ MT Prevalence } \\
\hline & Raw & True $(95 \% \mathrm{Cl})$ & Raw & True $(95 \% \mathrm{Cl})$ & Raw & True $(95 \% \mathrm{Cl})$ \\
\hline Kaniyambadi * & 3.23 & $3.95(2.34-5.12)$ & 6.45 & $7.36(5.73-8.60)$ & 3.23 & 3.01 (1.43-4.13) \\
\hline Latteri * & 23.53 & $30.44(28.11-32.73)$ & 11.77 & 14.55 (12.75-16.07) & 5.88 & $6.59(4.96-7.82)$ \\
\hline Serkadu * & 39.33 & $51.8(48.06-55.72)$ & 16.38 & $20.79(18.43-23.02)$ & 1.65 & $0.88(0.00-2.08)$ \\
\hline Sirukanchi * & 27.17 & $35.37(31.37-39.58)$ & 30.01 & $39.2(35.03-43.62)$ & 6.19 & 7.01 (4.70-9.26) \\
\hline Thimiri * & 42.42 & $55.97(52.38-59.92)$ & 3.04 & $2.75(1.12-3.97)$ & 3.04 & $2.75(1.22-3.97)$ \\
\hline Ussoor * & 6.90 & $7.97(6.08-9.56)$ & 3.45 & $3.31(1.60-4.65)$ & 3.45 & 3.31 (1.60-4.65) \\
\hline Vadakadapanthangal* & 25.02 & $32.46(28.54-36.58)$ & 4.14 & $4.24(2.16-6.26)$ & 8.37 & $9.96(7.38-12.53)$ \\
\hline Rural & 20.56 & $26.43(24.49-28.26)$ & 8.88 & $10.65(9.09-11.83)$ & 4.04 & $4.11(2.57-5.16)$ \\
\hline Alamelumangapuram & 5.56 & $6.16(4.52-7.39)$ & 2.77 & $2.39(0.80-3.52)$ & 2.77 & $2.39(0.80-3.52)$ \\
\hline Bagayam & 19.36 & $24.81(22.72-26.76)$ & 3.22 & $3(1.42-4.12)$ & 3.22 & $3(1.42-4.12)$ \\
\hline K.V.Kuppam & 30.43 & 39.77 (36.89-42.76) & 4.34 & $4.52(2.85-5.78)$ & 4.34 & $4.52(2.85-5.78)$ \\
\hline Kaspa & 3.04 & $2.75(1.09-4.01)$ & 3.04 & $2.75(1.09-4.01)$ & 3.04 & $2.75(1.09-4.01)$ \\
\hline Thottapalayam & 9.38 & $11.33(9.52-12.82)$ & 3.13 & $2.88(1.26-4.06)$ & 3.13 & $2.88(1.26-4.06)$ \\
\hline Thuthipattu & 29.41 & 38.39 (35.20-41.71) & 8.82 & $10.53(8.49-12.32)$ & 2.95 & $2.58(0.88-3.92)$ \\
\hline Arcot & 3.34 & $3.16(1.54-4.36)$ & 3.34 & $3.16(1.54-4.36)$ & 3.34 & $3.16(1.54-4.36)$ \\
\hline Urban & 13.59 & $17.02(15.36-18.39)$ & 3.65 & $3.58(2.05-4.62)$ & 3.25 & $3.04(1.50-4.07)$ \\
\hline Total (rural \& urban) & 20.90 & 26.89 (24.47-28.67) & 5.41 & $5.96(4.45-7.01)$ & 2.24 & $1.67(0.12-2.69)$ \\
\hline
\end{tabular}

Figure 1: Map of Tamil Nadu showing the Vellore district with the location of urban and rural primary sample collected.

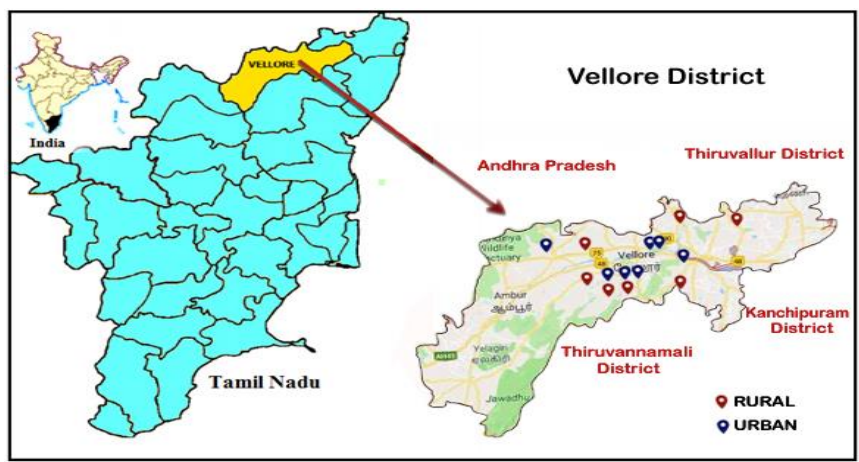

Figure 2: Overall seroprevalence of rickettsial infections in Vellore district, Tamil Nadu

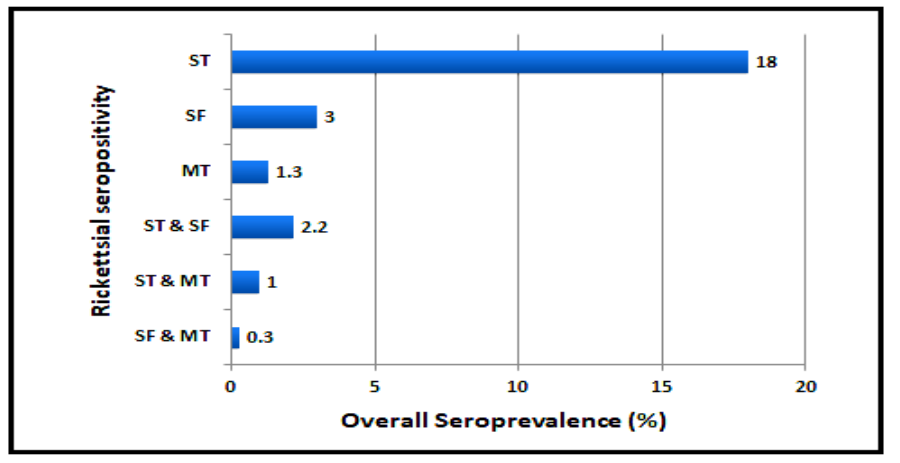




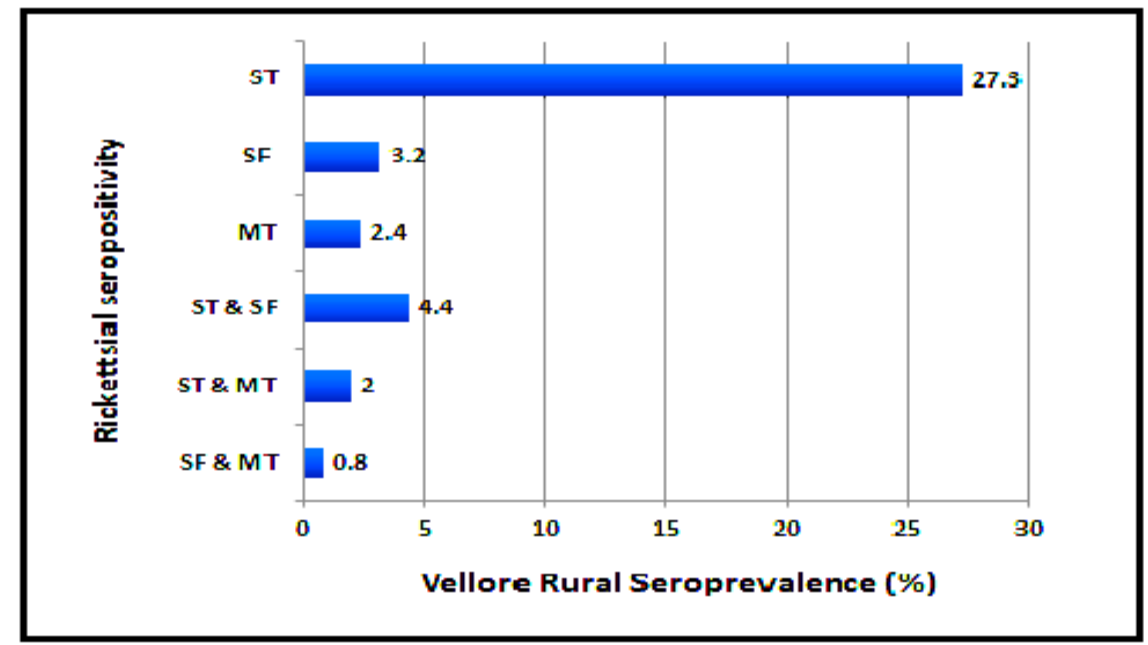

Figure 3: Prevalence of rickettsial seropositivity in Vellore district of Tamil Nadu. (Rural)

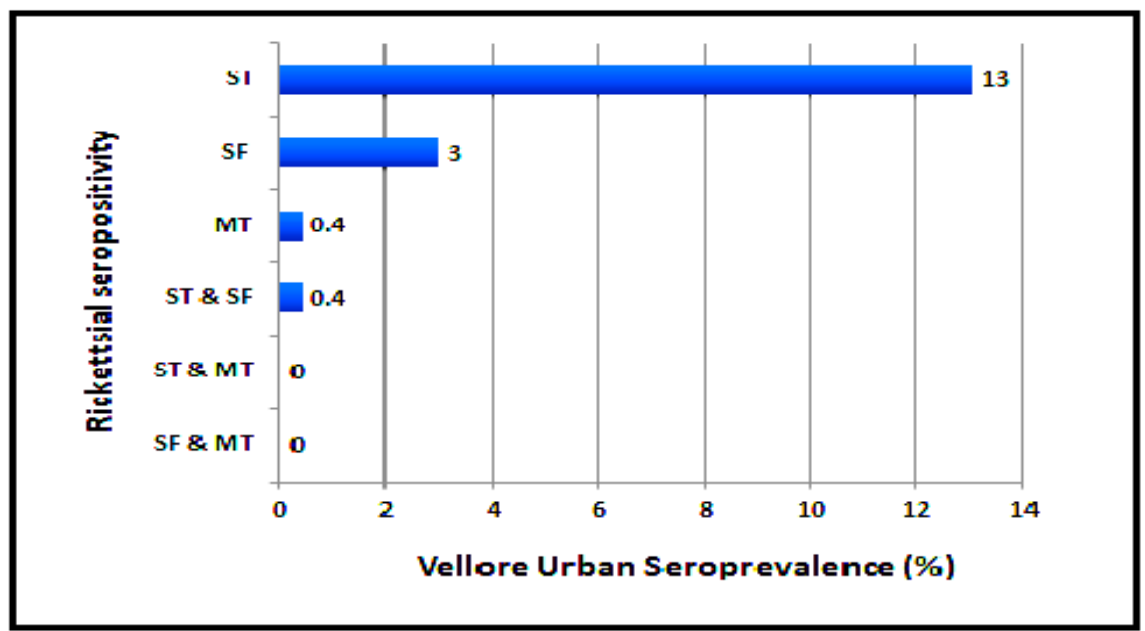

Figure 4: Prevalence of rickettsial seropositivity in Vellore district of Tamil Nadu. (Urban)

\section{DISCUSSION:}

Rickettsial infections are commonly recognized as an important cause of acute undifferentiated fever throughout India (10). The epidemiology, ecology, and clinical characteristics of the rickettsioses are highly variable and depend on geography (13).

This study demonstrated that almost $30 \%$ of the population of the Vellore district had previous exposure to rickettsial agents. Scrub typhus prevalence was highest followed by spotted fever and Murine typhus. Dual infections were also observed. Evidence of past exposure to two rickettsial agents was seen in $6.2 \%$ of the participants of which the majority were for scrub typhus and spotted fever (80\%). Cross-reactions between antibodies within the rickettsia species, especially between SF and MT, are known to occur. This is most likely due to multiple infections in an endemic setting or could be possible cross-reactions (14).

A study done in Vellore stated that the prevalence of scrub typhus is 20.3 in healthy individuals in rural Vellore (15) and 15\% among blood donors (16). The study by Devamani et al also reported the highest incidence of scrub typhus(20.4\%) followed by spotted fever(10.4\%) and murine typhus(5.4\%) among the south Indian population(10). Comparable results have been demonstrated in NE India by Khan et al(17) who reported seropositivity of $30.8 \%$, $13.8 \%$, and $4.2 \%$ among ST, SF, and MT group respectively, while by Mane et al (18) reported IgG seropositivity of $36.7 \%$ and $15.3 \%$ for SF and MT respectively from Gorakhpur, Uttar Pradesh. In south-east Asia, murine typhus was reported more in urban dwellers, while STG and SFG were more 
prevalent in rural dwellers (19). ST and SF were definitely more common in rural regions whereas murine typhus was marginally more common in rural areas of the Vellore district. The variation in prevalence among rural and urban areas may be due to the topographical differences of these areas. Limitations of this study were: Children were not included; longitudinal sampling was undertaken based on known hot spots of ST (derived from occurrence of 2 cases every year for the last 5 years). Future cohort studies that involve active fever surveillance will be more useful in assessing the prevalence.

\section{ACKNOWLEDGMENT:}

The authors gratefully acknowledge the Institutional Review Board of the Christian Medical College, Vellore (83-i/11/13) for approving the study and providing lab space and facilities.

\section{REFERENCES:}

1. Xu G, Walker DH, Jupiter D, Melby PC, Arcari CM. A review of the global epidemiology of scrub typhus. PLoS Negl Trop Dis. 2017 Nov 3; 11(11):e0006062.

2. Thiga JW, Mutai BK, Eyako WK, Ng'ang'a Z, Jiang J, Richards AL, et al. High Seroprevalence of Antibodies against Spotted Fever and Scrub Typhus Bacteria in Patients with Febrile Illness, Kenya. Emerg Infect Dis. 2015 Apr; 21(4):688-91.

3. Abdad MY, Abdallah RA, Fournier P-E, Stenos J, Vasoo S. A Concise Review of the Epidemiology and Diagnostics of Rickettsioses: Rickettsia and Orientia spp. J Clin Microbiol [Internet]. 2018 Aug 1 [cited 2020 Jul 20]; 56(8). Available from: https://jcm.asm.org/ content/56/8/e01728-17

4. Sahni SK, Narra HP, Sahni A, Walker DH. Recent molecular insights into rickettsial pathogenesis and immunity. Future Microbiol. 2013 Oct; 8(10):1265-88.

5. Izzard L, Fuller A, Blacksell SD, Paris DH, Richards AL, Aukkanit N, et al. Isolation of a Novel Orientia Species (O. chuto sp. nov.) from a Patient Infected in Dubai. J Clin Microbiol. 2010 Dec; 48(12):4404-9.

6. Robinson MT, Satjanadumrong J, Hughes T, Stenos J, Blacksell SD. Diagnosis of spotted fever group Rickettsia infections: the Asian perspective. Epidemiol Infect [Internet]. 2019 Oct 7 [cited 2020 Jul 20]; 147. Available from: https://www.ncbi.nlm.nih.gov/pmc/ articles/PMC6805790/

7. N R, A R. Rickettsial infections: an Indian perspective. Indian Pediatr. 2010 Feb 1; 47(2):157-64.
8. Vellore topographic map, elevation, relief [Internet]. Topographic-map.com. [cited 2020 Aug 6]. Available from: https://en-in.topographic-map.com/maps/g0by /Vellore/

9. Vellore District Population Census 2011-2020, Tamil Nadu literacy sex ratio and density [Internet]. [Cited 2020 Aug 6]. Available from: https://www.census 2011. co.in/census/district/23-vellore.html

10. Devamani CS, Schmidt W-P, Ariyoshi K, Anitha A, Kalaimani S, Prakash JAJ. Risk Factors for Scrub Typhus, Murine Typhus, and Spotted Fever Seropositivity in Urban Areas, Rural Plains, and Peri-Forest Hill Villages in South India: A Cross-Sectional Study. Am J Trop Med Hyg. 2020 Jul; 103(1):238-48.

11. Kohn JS Michael. Sample Size Calculators [Internet]. [Cited 2020 Aug 13]. Available from: https://samplesize.net/

12. Lang Z, Reiczigel J. Confidence limits for the prevalence of disease adjusted for estimated sensitivity and specificity. Prev Vet Med. 2014 Jan 1; 113(1):13-22.

13. Trung NV, Hoi LT, Thuong NTH, Toan TK, Huong TTK, Hoa TM, et al. Seroprevalence of Scrub Typhus, Typhus, and Spotted Fever Among Rural and Urban Populations of Northern Vietnam. Am J Trop Med Hyg. 2017 May; 96(5):1084-7.

14. Phommasone K, Paris DH, Anantatat T, CastonguayVanier J, Keomany S, Souvannasing $P$, et al. Concurrent Infection with Murine Typhus and Scrub Typhus in Southern Laos - the Mixed and the Unmixed. PLoS Negl Trop Dis. 2013 Aug 29; 7(8):e2163.

15. Hospitalizations and outpatient visits for undifferentiated fever attributable to scrub typhus in rural South India: Retrospective cohort and nested case-control study [Internet]. [Cited 2020 Aug 13]. Available from: https://journals.plos.org/plosntds/arti cle?id=10.1371/journal.pntd.0007160

16. Sengupta M, Anandan S, Daniel D, Prakash JAJ. Scrub Typhus Seroprevalence in Healthy Indian Population. J Clin Diagn Res JCDR. 2015 Oct; 9(10): DM01-2.

17. Khan SA, Bora T, Chattopadhyay S, Jiang J, Richards AL, Dutta P. Seroepidemiology of rickettsial infections in Northeast India. Trans R Soc Trop Med Hyg. 2016 Aug 1; 110(8):487-94.

18. Seroprevalence of spotted fever group and typhus group rickettsiae in individuals with acute febrile illness from Gorakhpur, India. Int J Infect Dis. 2019 Feb 1; 79:195-8.

19. Aung AK, Spelman DW, Murray RJ, Graves S. Rickettsial infections in Southeast Asia: implications for the local populace and febrile returned travellers. Am J Trop Med Hyg. 2014 Sep; 91(3):451-60. 